\title{
ARTICLES PUBLISHED ELSEWHERE \\ AGENT SEARCHING IN A TREE AND THE OPTIMALITY OF ITERATIVE DEEPENING
}

\author{
by P. Dasgupta, P.P. Chakrabarti, S.C. DeSarkar ${ }^{1}$
}

Kharagpur, India

Artificial Intelligence 71 (1994) 195-208

We reproduce the abstract:

The agent searching framework models the effort of a search strategy in terms of the distance traversed by an agent while exploring the search space. The framework has been found to be useful in modeling search problems where the cost of backtracking and retracing search paths is important in determining search complexity. In this paper we show that depth-first iterative deepening (DFID) strategies are optimal for an agent searching in a line, in $m$ concurrent rays, and in uniform $b$-ary trees. In the conventional search model it is known that DFID is asymptotically optimal for uniformed search of uniform $b$-ary trees. In this paper we prove the stronger result that for agent searching in uniform $b$-ary trees, iterative deepening is optimal up to lower-order terms. We also discuss the problems involved in optimally performing agent search in a graph.

\section{A BIBLIOGRAPHY ON MINIMAX TREES}

\section{Claude G. Diderich ${ }^{2}$}

Lausanne / Switzerland

We reproduce the abstract:

This technical report lists all theoretical and practical papers, theses and reports known to the author on the subject of studying minimax trees and analyzing sequential and parallel minimax tree search algorithms (some 175 papers).

\section{A SURVEY ON MINIMAX TREES AND ASSOCIATED ALGORITHMS}

\section{Claude G. Diderich and Marc Gengler ${ }^{2}$}

Lausanne / Switzerland

We reproduce the abstract:

This paper surveys theoretical results about minimax game trees and the algorithms used to explore them. The notion of game trees is formally introduced and its relation with game playing described. The first part of the survey outlines major theoretical results about minimax game trees, their size and the structure of their subtrees. In the second part of this paper, we survey the various sequential algorithms that have been developed to explore minimax trees. The last part of this paper tries to give a succinct view on the state of the art in parallel minimax game tree searching.

1 Department of Computer Science and Engineering, Indian Institute of Technology, Kharagpur, India 721302. Email: ppchack@cse.iitkgp.ernet.in.

2 Ecole Polytechnique Federale de Lausanne, Laboratoire d'Informatique Theorique, IN (Ecublens), CH-1015 Lausanne, Switzerland. Email: diderich@.di.epfl.ch. 